\title{
Neural Basis of Adaptive Response Time Adjustment during Saccade Countermanding
}

\author{
Pierre Pouget, ${ }^{1}$ Gordon D. Logan, ${ }^{1}$ Thomas J. Palmeri, ${ }^{1}$ Leanne Boucher, ${ }^{1}$ Martin Paré, ${ }^{2}$ and Jeffrey D. Schall ${ }^{1}$ \\ ${ }^{1}$ Center for Integrative \& Cognitive Neuroscience, Vanderbilt Vision Research Center, Department of Psychology, Vanderbilt University, Nashville, \\ Tennessee 37240, and ${ }^{2}$ Centre for Neuroscience Studies, Queen's University, Kingston, Ontario, Canada K7L 3N6
}

Humans and macaque monkeys adjust their response time adaptively in stop-signal (countermanding) tasks, responding slower after stop-signal trials than after control trials with no stop signal. We investigated the neural mechanism underlying this adaptive response time adjustment in macaque monkeys performing a saccade countermanding task. Earlier research showed that movements are initiated when the random accumulation of presaccadic movement-related activity reaches a fixed threshold. We found that a systematic delay in response time after stop-signal trials was accomplished not through a change of threshold, baseline, or accumulation rate, but instead through a change in the time when activity first began to accumulate. The neurons underlying movement initiation have been identified with stochastic accumulator models of response time performance. Therefore, this new result provides surprising new insights into the neural instantiation of stochastic accumulator models and the mechanisms through which executive control can be exerted.

\section{Introduction}

Stochastic accumulator models provide a powerful explanation of the random and systematic variation of response times (RTs) in speeded decision-making tasks (Smith and Ratcliff, 2004; Bogacz et al., 2006; Gold and Shadlen, 2007). They account for RT and choice probability in terms of three basic parameters: baseline activity before accumulation begins, the rate of accumulation once it begins, and the threshold for terminating the decision process. A fourth parameter, residual processing time, includes the time between the presentation of a stimulus and the beginning of accumulation plus the time between reaching a threshold and executing the response. The neural instantiation of the processes described by these models was first elucidated with the discovery that responses are initiated when the random accumulation of activity in particular movement-related neurons reaches

Received April 14, 2011; revised June 24, 2011; accepted July 14, 2011.

Author contributions: G.D.L., M.P., and J.D.S. designed research; M.P. and J.D.S. performed research; P.P., G.D.L., T.J.P., L.B., M.P., and J.D.S. analyzed data; P.P., G.D.L., T.J.P., L.B., M.P., and J.D.S. wrote the paper.

This work was supported by NIH Grants R01-MH55806, P30-EY08126, and P30-HD015052, Air Force Office of Scientific Research Grant FA 9550-07-1-0192, NSF Grants SBE-0542013 and BCS 0957074, the Temporal Dynamics of Learning Center SBE-0542013, an NSF Science of Learning Center, the Canadian Institutes of Health Research, and the EJLB Foundation. Support was also provided by Robin and Richard Patton through the E. Bronson Ingram Chair of Neuroscience. M.P. holds an Early Researcher Award from the Ontario Ministry of Research and Innovation. We thank Robert H. Wurtz (National Eye Institute) for his generous support, John Haitas for help with the analysis and simulations, Mike Shadlen and Roger Carpenter for discussion and helpful comments on the manuscript, and the Vanderbilt Advanced Center for Computing for Research and Education for access to the high-performance computing cluster.

P. Pouget's present address: CRICM, INSERM UMRS 975, CNRS UMR 7225, UPMC, 47 Boulevard de I'Hôpital, NEB, Hospital de la Salpetriere, 75013 Paris, France.

L. Boucher's present address: Farquhar College of Arts and Sciences, Division of Social and Behavioral Sciences, Nova Southeastern University, Fort Lauderdale, FL 33321.

Correspondence should be addressed to Dr. Jeffrey D. Schall, Department of Psychology, Vanderbilt University, PMB 407817, 2301 Vanderbilt Place, Nashville, TN 37240-7817. E-mail: jeffrey.d.schall@vanderbilt.edu; or Dr. Martin Paré, Centre for Neuroscience Studies, Queen's University, Botterell Hall, Room 438, Kingston, Ontario, Canada K7I3N6, E-mail: martin.pare@queensu.ca.

DOI:10.1523/JNEUROSCI.1868-11.2011

Copyright $\odot 2011$ the authors $\quad 0270-6474 / 11 / 3112604-09 \$ 15.00 / 0$ a fixed threshold (Hanes and Schall, 1996). Subsequently, several investigators have shown how the activity before choice responses of particular neurons in posterior parietal cortex, frontal eye field, and the superior colliculus can be identified quantitatively with the stochastic accumulation process described by race and diffusion models (Mazurek et al., 2003; Ratcliff et al., 2007; Purcell et al., 2010).

Stochastic accumulator models account for adaptation of RT to minimize errors and maximize rewards. This adaptation is commonly supposed to be accomplished through changes in the threshold of accumulation that triggers a response (Nakahara et al., 2006; Simen et al., 2006; Schall and Boucher, 2007; Forstmann et al., 2008; Ratcliff and McKoon, 2008). The hypotheses that adaptation of RT is accomplished through changes in the threshold of accumulation has not been tested directly by measurements in neurons identified with the parameters of stochastic accumulator models in monkeys optimizing RT to perform a task.

We addressed this question using data from macaque monkeys performing a saccade stop-signal (countermanding) task (Logan and Cowan, 1984; Hanes et al., 1998; Paré and Hanes, 2003; Brown et al., 2008). Humans and macaque monkeys adapt their performance in stop-signal tasks, producing longer RTs after successfully inhibiting a planned movement (Rieger and Gauggel, 1999; Emeric et al., 2007; Verbruggen and Logan, 2008c; Nelson et al., 2010). These data provide a unique opportunity to determine whether the neural implementation of systematic adjustments of RT is accomplished through a change of threshold, baseline, or accumulation rate or alternatively through a change in the onset time of accumulation. Our analysis is based on the identification of presaccadic movement-related activity with the accumulation process described by a stochastic accumulator model of stop-signal performance (Boucher et al., 2007). Several lines of evidence support this linking proposition (Schall, 2004). 


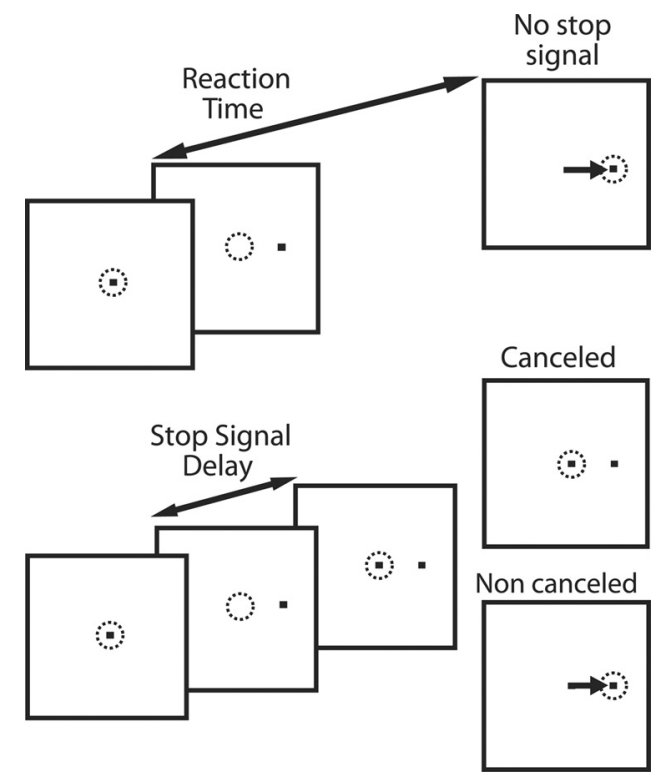

Figure 1. Saccade stop-signal (countermanding) task. Dotted circles indicates gaze position, and the arrow indicates the direction of the saccade. All trials began with the presentation of a central fixation spot. After a variable fixation interval, the fixation spot disappeared and, simultaneously, a target appeared at an eccentric location to the right or left of central fixation. On $\sim 30 \%$ of trials (stop-signal trials), the fixation spot was reilluminated after an interval (stopsignal delay) after target onset. Fixation reillumination was the cue for the monkeys to withhold a saccade to the target. Trials in which monkeys were successful in maintaining fixation were referred to as canceled trials, and trials in which monkeys made a saccade to the eccentric target were referred to as noncanceled trials. For the remaining $\sim 70 \%$ of trials (n0-stop-signal trials), the fixation point was not reilluminated and monkeys were reinforced for making a saccade to the peripheral target.

First, the activity of these neurons in frontal eye fields (FEFs) and superior colliculus (SC) is modulated in a manner sufficient to control the initiation of eye movements in the stop-signal task. Saccades are initiated when the activity of these neurons reaches a threshold of discharge rate, and the variability in $\mathrm{RT}$ across trials arises from stochastic variation in the rate at which the activity accumulates to the threshold (Sparks, 1978; Hanes and Schall, 1996; Dorris et al., 1997). Thus, neural measures of the onset of accumulation, baseline discharge rate before the onset, rate of accumulation, and threshold can be identified with the corresponding parameters in a stochastic accumulator model.

\section{Materials and Methods}

Methods for collecting and analyzing the performance and neurophysiological data have been described in detail previously (Hanes et al., 1998; Paré and Hanes, 2003). Here we will summarize the basic elements of the task and emphasize the new approach taken with this dataset.

Stop-signal task performance measures. On no-stop-signal trials, monkeys were reinforced for shifting gaze to a target that appeared when the fixation spot disappeared (Fig. 1). On stop-signal trials, the fixation spot reappeared after a variable delay following target onset. The stop-signal delays ranged from 25 to $275 \mathrm{~ms}$ for the FEF data and from 25 to $230 \mathrm{~ms}$ for the SC data; the values were selected from one of four values with intervals of 40,50 , or $60 \mathrm{~ms}$ adjusted for each session such that at the shortest delay monkeys withheld the movement on $>85 \%$ of trials and at the longest delay monkeys withheld the movement on $<15 \%$ of trials. Monkeys were reinforced if they withheld the planned saccade (referred to as "signal-inhibit" or "canceled" trials) but not if they failed to inhibit the saccade (referred to as "signal-respond" or "noncanceled" trials). The percentage of trials on which a stop signal occurred ranged from $30 \%$ to $50 \%$, and none of the findings varied with this quantity. For each data collection session, saccade RT and associated neuronal activity on no- stop-signal trials were sorted as a function of whether the preceding trial was a no-stop-signal or canceled trial. RT was measured in no-stopsignal trials preceded by another no-stop-signal trial or preceded by a stop-signal trial in which monkeys inhibited the response. In previous work, we have found that RT measures longer on trials following successful inhibition but less, if at all, after unsuccessful inhibition (Emeric et al., 2007; see also Nelson et al., 2010).

Data were selected on the basis of the quality of task performance and the characteristics of the neural activity. Criteria for task performance were sufficient numbers of each type of trial $(>10)$ and probability of failing to stop ranging from $<20 \%$ at the shortest stop-signal delay to $>80 \%$ at the longest stop-signal delay.

Neuron functional classification. FEF and SC consist of a diversity of neurons that exhibit qualitatively different patterns of modulation in this task (Hanes et al., 1998; Paré and Hanes, 2003; Ray et al., 2009; see also Murthy et al., 2009). Our analyses focused on movement-related neurons and visually responsive neurons. These different types of neurons were distinguished using multiple criteria. These criteria are quantitative extensions of conventional criteria used to distinguish functional classes of neurons in visuomotor structures like FEF and SC.

One set of classification criteria were applied to data obtained during a memory-guided saccade task in which monkeys direct gaze to a fixation spot and maintain fixation after a peripheral target is flashed. A gaze shift to the remembered location can be initiated only after the fixation spot is removed. This procedure distinguishes visually evoked from movementrelated activity. In the memory guided saccade task, movement neurons exhibit an accumulation of activity only before saccade initiation, while visually responsive neurons exhibit a consistent visual response with a latency $<100 \mathrm{~ms}$ following target onset.

The stop-signal task provides additional classification criteria. Whereas movement neurons exhibit a pronounced modulation of discharge rate on canceled stop-signal trials coincident with stop-signal reaction time (SSRT), visual neurons exhibit either no modulation at all or a modulation well after SSRT on canceled stop-signal trials (Hanes et al., 1998; Paré and Hanes, 2003; Brown et al., 2008). Another recent analysis has demonstrated that visuomovement neurons are clearly distinguished from movement neurons by the timing and pattern of their modulation (Ray et al., 2009). Only movement neurons exhibit the progressive accumulation of activity that corresponds to stochastic accumulation, like that seen in the activation of the GO units in the interactive race model (Boucher et al., 2007).

On the basis of data obtained in the stop-signal task, movement neurons that met the following criteria were selected: (1) differential rate of growth of movement activity for short versus long RT on no-stop-signal trials; (2) negative modulation of discharge rate on canceled stop-signal trials occurring before stop-signal reaction time; (3) progressively higher discharge rate on canceled stop-signal trials with longer stop-signal delays; and (4) no visual responses or only small visual transients followed by a return of discharge rate below or near baseline activity before the beginning of accumulation of movement activity; the average rate at onset time was 10 and 5 spikes/s for SC and FEF neurons, respectively. Visually responsive neurons met the following criteria: (1) consistent visual response with a latency $<100 \mathrm{~ms}$ and (2) absence of modulation before the RT to the stop signal on canceled stop-signal trials. These neurons included both visual neurons, which were characterized by an absence of modulation of discharge rate before stop-signal reaction time in canceled trials, and visuomovement neurons, which showed no differential rate of growth of movement activity for short versus long RT on no-stop-signal trials (Ray et al., 2009).

Measures of neural activity. We examined the average neuronal activity measured during no-stop-signal trials as a function of whether the preceding trial was a no-stop-signal trial or a canceled stop-signal trial. Neural activity was measured through spike density functions constructed by convolving spike trains with a filter shaped like a postsynaptic potential (Hanes et al., 1998; Paré and Hanes, 2003). Our analyses focused on four characteristics of the activity of movement-related neurons that could contribute to the observed variation of RT: differences in the baseline firing rate, the threshold activation level at which saccades were initiated, the rate of accumulation of activation to the threshold, and the 
Table 1. Observed parameters

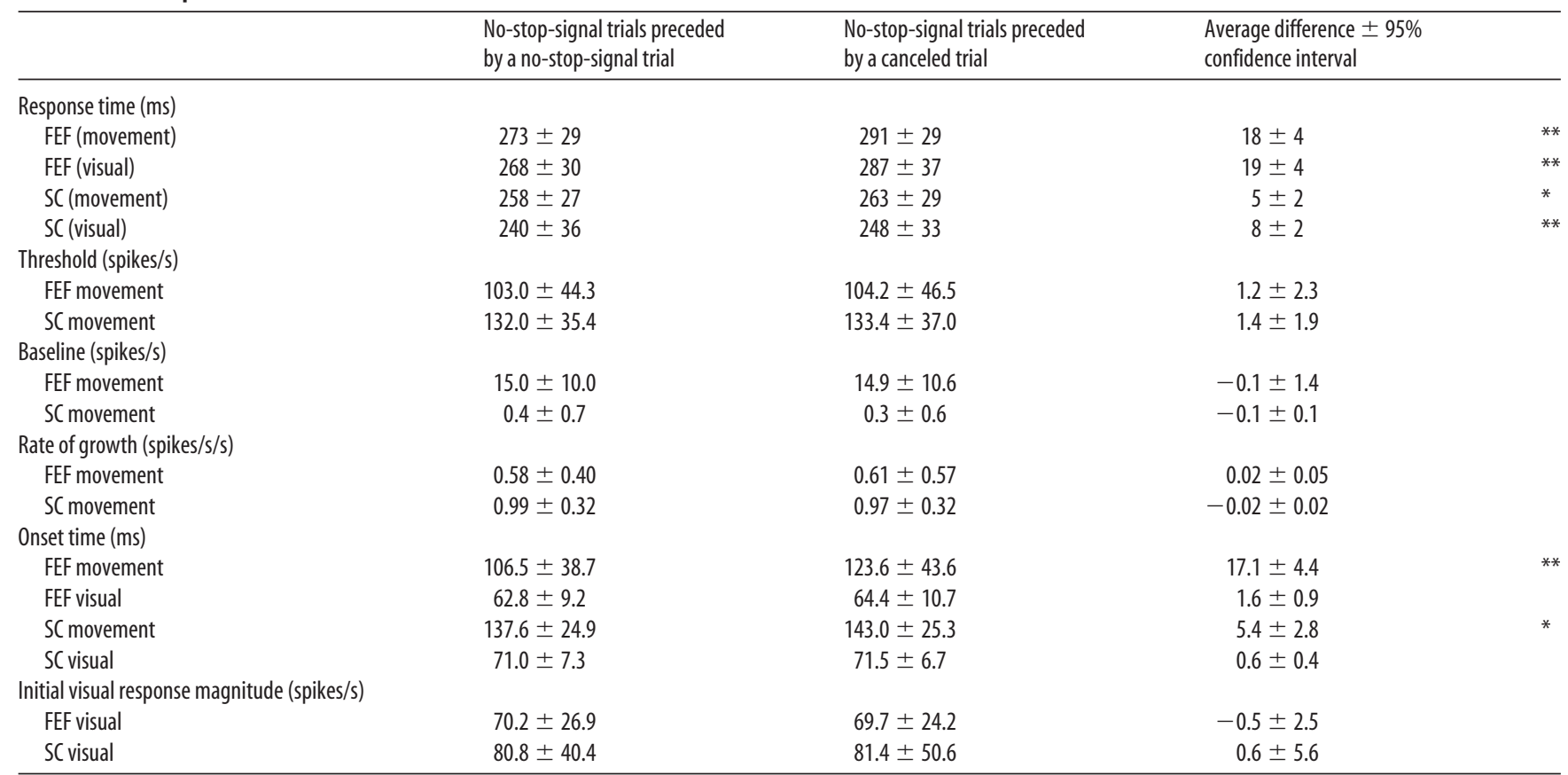

All trials were included in the calculation of response time; only trials in which the target fell in the neuron's response field were used to calculate the indicated parameters. Data in the first two columns are mean $\pm S D$. ${ }^{*} p<$ $0.05,{ }^{* *} p<0.01$.

onset of movement-related activity. Baseline activity was measured as the average discharge rate from $-200 \mathrm{~ms}$ until the target appeared. Threshold activation was measured as the average discharge rate in the interval 10-20 ms before saccade initiation (Hanes and Schall, 1996). The onset of movement-related activity was measured with a sliding-window algorithm working backward from the peak of activity of movement neurons relative to target onset (Woodman et al., 2008). The onset of activity was defined as the first time point that met the following criteria: the spike density function did not increase significantly according to a Spearman correlation $(\alpha=0.05)$ over a time window ranging from $-10 \mathrm{~ms}$ to +10 $\mathrm{ms}$, the correlation between spike density and time remained nonsignificant for $10 \mathrm{~ms}$ as the center of the window continued to move backward from the saccade; if any of the values of the spike density function within $10 \mathrm{~ms}$ forward of the onset time identified by the correlation algorithm fell below the activity level at that onset time, the onset time was then redefined as the time point at which the spike density function was lowest. For four of the movement neurons recorded in FEF, the number of trials and the spread of the RT did not permit use of this method, so the algorithm was modified to operate from the time of saccade initiation. We verified that adjustment of the algorithm did not bias the measurement of onset time.

The rate of accumulation was measured three ways because its value depends on the other parameters. One measure of rate was simply the difference between the threshold and baseline discharge rate divided by the difference between RT and the onset of accumulation. Another measure was the difference between the threshold discharge rate and the discharge rate at the onset of accumulation divided by the difference between RT and the onset of accumulation. A third method that did not depend on the measurement of the onset of accumulation was the difference in discharge rates when the activity was at $70 \%$ of threshold and when it was at $30 \%$ of threshold divided by the time when activity reached $70 \%$ of threshold and the time when activity reached $30 \%$ of threshold. Each measure was determined for each neuron for the collection of no-stop-signal trials preceded by either a no-stop-signal trial or by a canceled stop-signal trial.

Other measures were used to characterize the activity of visually responsive neurons. The onset time of the initial visual response was defined as the time at which the activity of visual neurons exceeded their mean baseline activity level by $5 \mathrm{SDs}$. The initial visual response magni- tude was taken as the mean spike density function during the first $25 \mathrm{~ms}$ of the response.

Linear ballistic accumulator model. The linear ballistic accumulator model assumes between-trial noise such that the rate on a simulated trial is drawn from a normal distribution with mean given by the mean rate and standard deviation $\sigma$ (Brown and Heathcote, 2008). We used a $1 \mathrm{~ms}$ time step and set $\sigma=2.5$ units. Note that the units for noise, as well as the units for rate and threshold, are arbitrary. Based on parameters of onset, rate, and threshold, we generated predicted RT distributions using Monte Carlo simulations of 5000 trials per distribution. For data from each session, best-fitting parameter values for rate, threshold, and onset were found using the subplex algorithm (Rowan, 1990), a variant of the Nelder-Mead simplex method (Nelder and Mead, 1965) that is well suited for optimizing stochastic models. Like most hill-climbing parameter search algorithms, subplex is susceptible to hitting local minima, so we started the subplex algorithm with at least 160 different starting points in parameter space for each dataset we fit. Following Ratcliff and Tuerlinckx (2002), we optimized the fit of each model to the behavioral data by minimizing a Pearson $\left(\chi^{2}\right)$ statistic,

$$
\chi^{2}=\sum_{i} \frac{\left(o_{i}-p_{i}\right)^{2}}{p_{i}} .
$$

In this case, observed $\left(o_{i}\right)$ and predicted $\left(p_{i}\right)$ refer to the number of trials with RTs falling into each quintile bin of the observed and predicted RT distribution. Simulated trials were rescaled so that that total number of model trials equaled the total number of observed trials. After the models were fit to behavioral data from each session with FEF or SC recordings, we tested for significant differences in onset, rate, and threshold using a standard $t$ test on the best-fitting parameter values. Note that whereas the data presented in Table 1 used only trials in which the target fell in the neurons' movement or receptive field, the data used for the model fits included trials at all target locations.

\section{Results}

The major innovation of this study was to analyze the temporal pattern of activity contingent on the history of behavioral performance. The results reported here are based on a reanalysis of data 

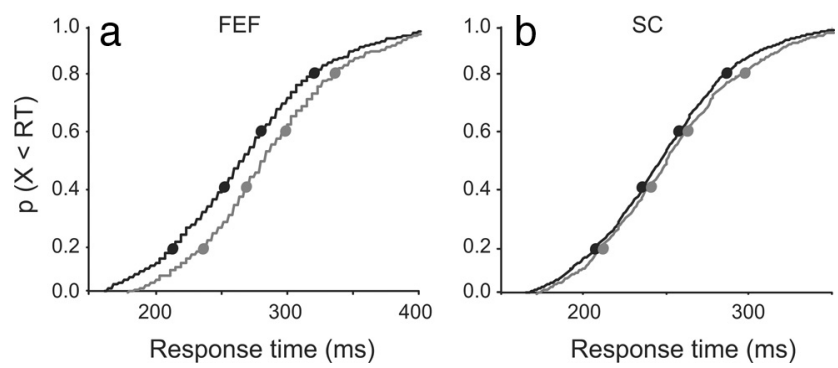

Figure 2. Vincentized response time distributions for no-stop-signal trials preceded by either a no-stop-signal trial (black line) or a canceled trial (gray line) for data collected in sessions during which movement cells were recorded in $\mathrm{FEF}(\boldsymbol{a})$ and SC $(\boldsymbol{b})$.

collected previously. The data for this study were derived from a subset of the single-unit recordings and performance measures from macaque monkeys performing a saccade stop-signal task that have been reported before; data were collected from the FEF of two male monkeys (Hanes et al., 1998) and from the intermediate and deep layers of the SC of two other male monkeys (Paré and Hanes, 2003). In total, 36 movement neurons (22 from FEF and 14 from SC) and 48 visually responsive neurons (26 from FEF and 22 from SC) met the criteria describe above for these analyses. The data from the movement neurons in the FEF consisted of a total of 756 trial sequences in which a no-stop-signal trial followed a no-stop-signal trial (nostop-nostop) and 327 trial sequences in which a no-stop-signal trial followed a canceled stop-signal trial (canceled-nostop), with averages of 34 and 15 trials per neuron, respectively. The data from the movement neurons in the SC consisted of a total of 5478 nostop-nostop trial sequences and 1436 canceled-nostop trial sequences, with averages of 391 and 103 trials per neuron, respectively.

\section{Systematic variation of RT with trial history}

Overall across the 82 sessions in which both movement and visual neurons were recorded from the FEF and the SC, saccade RTs on no-stop-signal trials preceded by a canceled trial were significantly longer than RTs on no-stop-signal trials preceded by a no-stop-signal trial ( 260 vs $274 \mathrm{~ms}, t$ test $p<0.001$ ) (Fig. 2, Table 1). This RT difference happened to be larger in sessions when FEF neurons were recorded than in sessions when SC neurons were recorded (FEF monkey $1-276$ vs $297 \mathrm{~ms}, p<0.001$; FEF monkey $2-252$ vs $266 \mathrm{~ms}, p=0.025$; SC monkey $1-229$ vs $236 \mathrm{~ms}, p<$ 0.001 ; SC monkey $2-268$ vs $274 \mathrm{~ms}, p=0.03$ ). The data from the two structures were obtained in different monkeys in different laboratories. The RT difference was smaller for the SC data, probably because these monkeys performed many more trials than did the monkeys providing the FEF data. Ultimately, the same qualitative optimization of performance was observed during collection of both FEF and SC data, constituting independent replications of the phenomenon. The quantitative differences in RT between FEF and SC sessions are not pertinent to this study, for it does not aim to compare FEF and SC neuronal activity.

Because RT measured on no-stop-signal trials following unsuccessful inhibition trials was elevated little if at all, neurophysiological data from these trials is not useful for evaluating the mechanism of adaptive adjustment. Moreover, because stop trials in which inhibition was unsuccessful were not reinforced, RT analyses of the sequences involving these trials could be confounded.

\section{Pattern of activity of movement neurons}

Individually (Fig. 3) and collectively (Fig. 4) across the population of movement neurons in FEF and SC, saccades were initiated

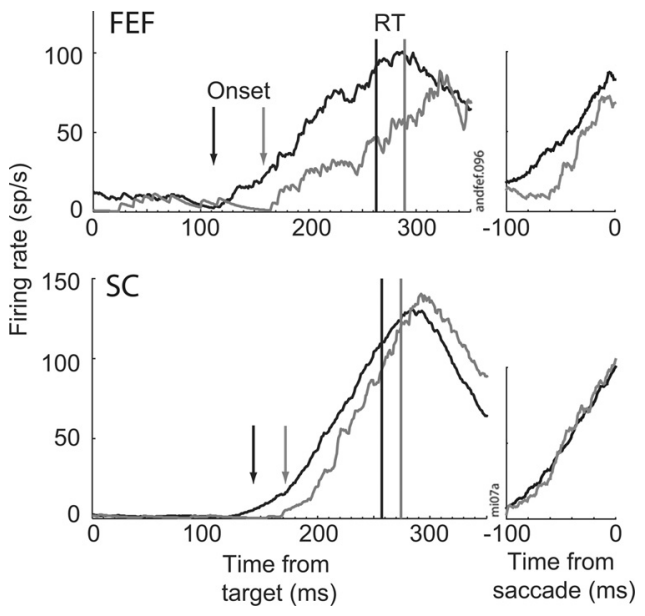

Figure 3. Time course of activity of representative movement neuron in FEF (top) and in SC (bottom) aligned on target presentation (left) and saccade initiation (right) for no-stop-signal trials preceded by either no-stop-signal (black) (FEF $n=34$, SC $n=371$ ) or canceled stopsignal (gray) ( $F E F n=9, S C n=73$ ) trials. The onset (vertical arrow) and average RT (vertical line) are indicated for both neurons for both trial sequences.
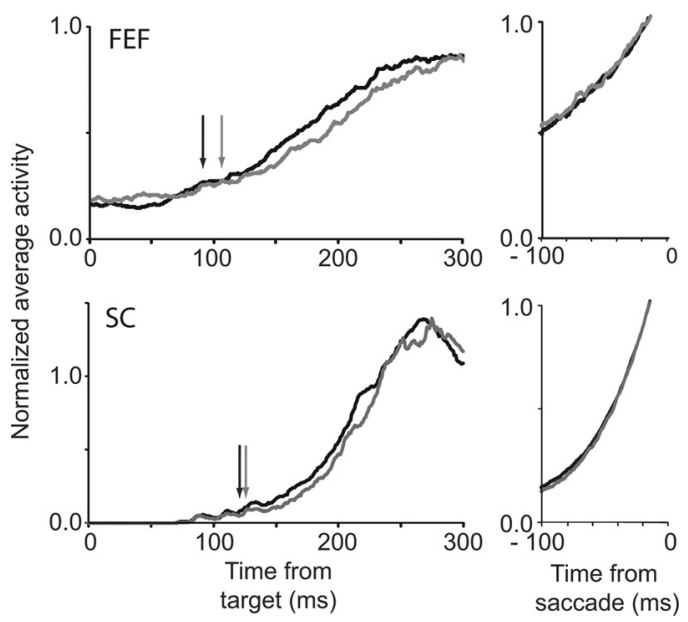

Figure 4. Time course of grand average of activity of FEF (top) and SC (bottom) neurons aligned on target presentation (left) and saccade initiation (right) for no-stop-signal trials preceded by either no-stop-signal (black) or canceled stop signal (gray). The discharge rates were normalized to the trigger thresholds of individual neurons. Average onset times indicated by the vertical lines. Note the common thresholds and rates of accumulation, especially in the plots aligned on saccade initiation. Also note the continuously earlier accumulation in NS-NS as compared to C-NS trials.

when the activity reached a threshold that did not vary significantly with RT. The random variability of RT within an experimental condition arose from stochastic variation in the rate of accumulation of the discharge rate to the threshold (Sparks, 1978; Hanes and Schall, 1996; Dorris et al., 1997). On successful inhibition trials, activity in the same movement neurons begins to increase and then abruptly decreases before reaching threshold, so no response is triggered (Hanes et al., 1998; Paré and Hanes, 2003). The shift from increasing to decreasing firing rate occurs before the SSRT, so these neurons can be in the causal chain controlling initiation of the response.

To accomplish the systematic delay of RT after successful inhibition, the presaccadic movement activity could vary in one of four respects: increasing threshold, decreasing baseline, reducing rate of accumulation, or delaying the onset of accumulation after target appearance. We measured each of these parameters in the 

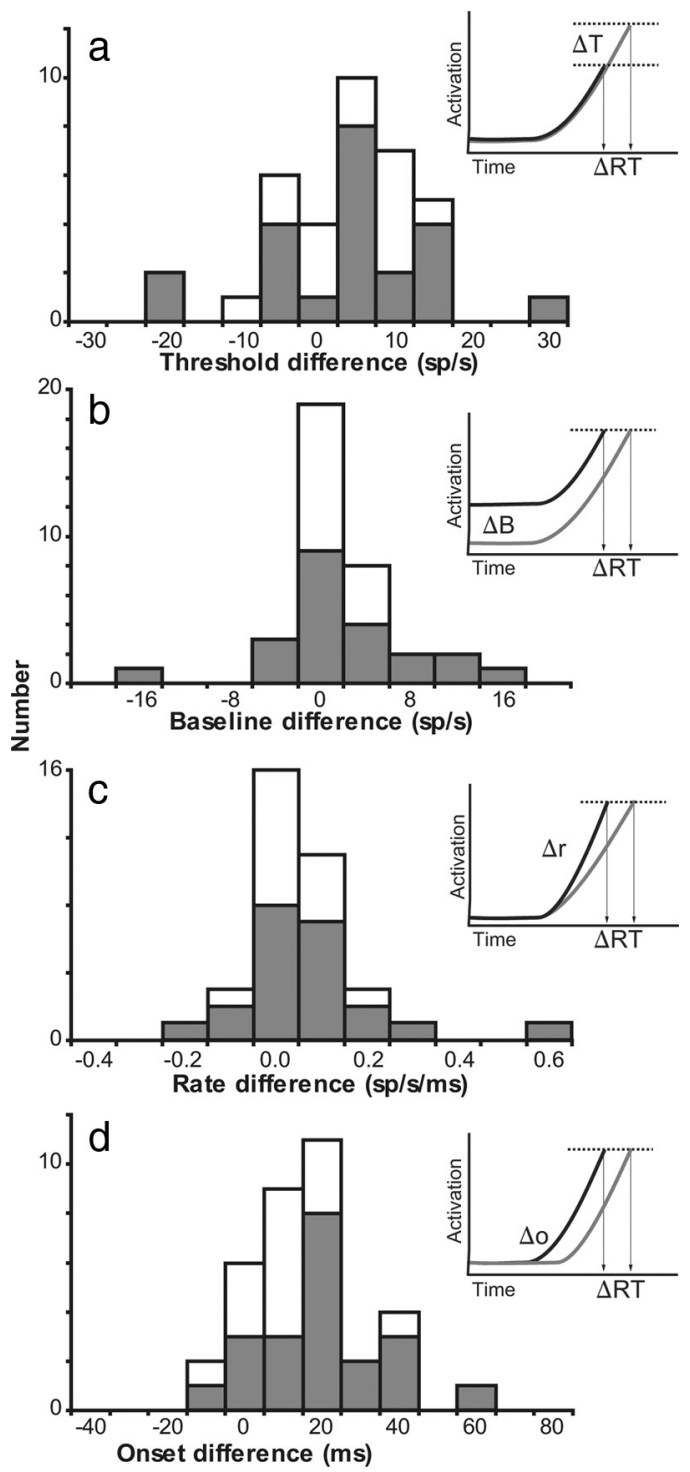

Figure 5. $\boldsymbol{a}-\boldsymbol{d}$, Stacked bar plots of the differences in the threshold activity level $(\Delta T)(\boldsymbol{a})$, baseline activity level $(\Delta B)(\boldsymbol{b})$, rate of growth $(\Delta r)(\boldsymbol{c})$, and time of onset of accumulation $(\Delta 0)$ (d) for FEF (filled) and SC (open) between no-stop-signal trials preceded by either a no-stopsignal trial or a canceled trial. Insets diagram the alternative mechanisms of RT change for no-stop-signal trials preceded by either a no-stop-signal trial (black) or a canceled trial (gray). Only the difference in time of onset of accumulation accounted for the RT difference ( $\Delta R T)$.

activity of each movement-related neuron recorded in no-stopsignal trials preceded by a no-stop-signal trial (faster RT condition) and in no-stop-signal trials preceded by a canceled trial (slower RT condition). Figure 5 and Table 1 summarize the main findings. In keeping with other quantitative neurophysiology studies, our conclusions are based on the central tendencies of the distributions of the values for each FEF and SC neuron. Obviously, these measurements have noise. Previous studies from this laboratory have examined the noise inherent in these measurements of neural modulation time and magnitude and demonstrated that conclusions derived from central tendencies are not contradicted when intrinsic noise is accounted for (e.g., Bichot et al., 2001; Sato and Schall, 2003; Brown et al., 2008).

We observed no significant increase in average threshold (Fig. $5 a$ ) and no significant decrease in average baseline (Fig. 5b). If, as predicted by most models, a change in threshold is the basis for the change in RT, then assuming no change of any other param- eter, the observed difference in threshold we measured would produce a $2 \mathrm{~ms}$ increase in RT for the FEF sessions and a $1 \mathrm{~ms}$ increase for the SC sessions. To account for the difference of RT measured during FEF recordings, the threshold of FEF movement neurons must increase eightfold more than it actually does. To account for the difference of RT measured during SC recordings, the threshold of SC movement neurons must increase fourfold more than it actually does. This analysis demonstrates conclusively that adjustments of threshold necessary to produce the observed changes of RT do not occur in FEF and SC movement neurons. The same argument holds for changes of baseline.

We also found no significant decrease in average rate of accumulation (Fig. $5 c$ ). The rate of accumulation was measured in multiple ways. Because RT = onset + (threshold - baseline)/ rate, each of the quantities we report are related. Table 1 reports a calculation of rate that afforded self-consistent values of RT, onset, baseline, and threshold.

If a change in rate of accumulation is the basis for the change in $\mathrm{RT}$, then assuming no change of any other parameter, the observed difference in rate we measured would produce a $5 \mathrm{~ms}$ decrease of RT for the FEF sessions and a $3 \mathrm{~ms}$ increase for the SC sessions. To account for the difference of RT measured during FEF recordings, the rate of accumulation of FEF movement neurons must decrease by $10 \%$ when the measured value was an increase of $3 \%$. To account for the difference of RT measured during SC recordings, the rate of accumulation of SC movement neurons must decrease twofold more than it actually does. This analysis demonstrates that adjustments of accumulation rate necessary to produce the observed changes of RT do not occur in FEF and SC movement neurons.

Because rate is in the denominator, small measurement errors can translate into large variation in RT. We measured the rate of accumulation in two other ways. First, we calculated rate $=$ (threshold - activity at onset time)/(RT - onset time). Second, we calculated rate $=$ (activity at $70 \%$ of threshold - activity at $30 \%$ of threshold)/(time when activity reached $70 \%$ of threshold - time when activity reached $30 \%$ of threshold). Table 2 lists the central tendencies of each of these values. Neither of the measures exhibited a significant difference that could account for the systematic change of RT with trial history. To summarize, these data illustrate the challenge in measuring the rate of accumulation in neural data and demonstrate that, in these data, no measurement provided evidence that the rate of accumulation could explain the systematic variation in RT.

In contrast, the average onset of accumulation for no-stop-signal trials preceded by no-stop-signal trials occurred significantly earlier than the average onset of accumulation in no-stop-signal trials preceded by canceled trials (Fig. $5 d$ ).

If a change in the onset of accumulation is the basis for the change in RT, then assuming no change of any other parameter, the observed difference in onset that we measured would produce a $17 \mathrm{~ms}$ increase of RT for the FEF sessions and a $5 \mathrm{~ms}$ increase for the SC sessions. These values correspond precisely to the observed $17 \mathrm{~ms}$ increase in RT for the FEF sessions and the $5 \mathrm{~ms}$ increase for the SC sessions.

These results indicate that the systematic variation in RT can be accounted for by variation of the onset of accumulation, but the data cannot rule out conclusively that trade-offs between parameters do not contribute some measure of variation as well. However, if such trade-offs occur, then it is hard to understand how the accumulation onset time could correspond so well to the observed difference in RT. 


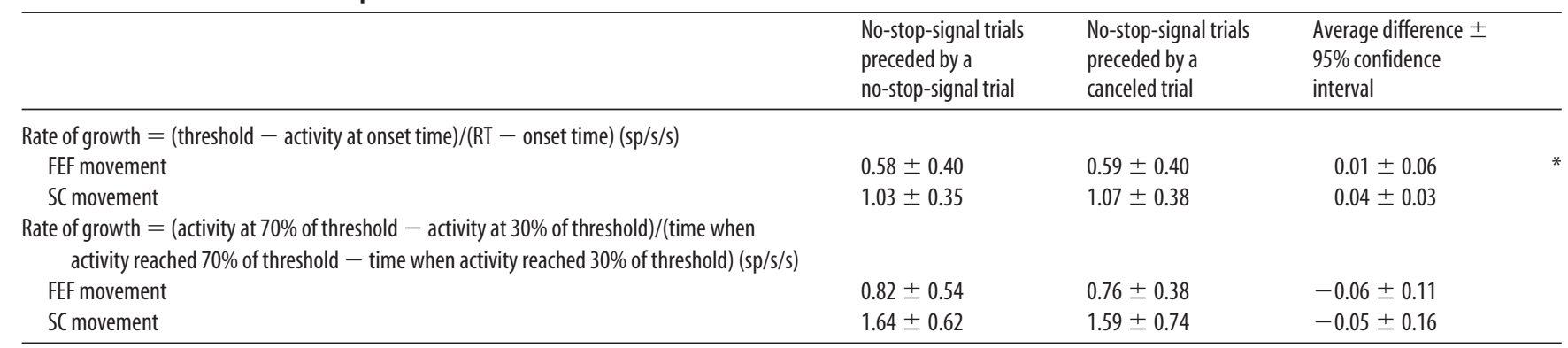

Data in the first two columns are mean \pm SD.

Table 3. Ballistic Accumulator Model parameters

\begin{tabular}{|c|c|c|c|c|}
\hline & $\begin{array}{l}\text { No-stop-signal trials } \\
\text { preceded by a } \\
\text { no-stop-signal trial }\end{array}$ & $\begin{array}{l}\text { No-stop-signal trials } \\
\text { preceded by a } \\
\text { canceled trial }\end{array}$ & $\begin{array}{l}\text { Average difference + } \\
95 \% \text { confidence } \\
\text { interval }\end{array}$ & \\
\hline \multicolumn{5}{|c|}{ Response time (ms) } \\
\hline FEF & $256 \pm 31$ & $274 \pm 26$ & $17.8 \pm 9.3$ & $* *$ \\
\hline SC & $243 \pm 31$ & $246 \pm 32$ & $3.2 \pm 4.4$ & * \\
\hline \multicolumn{5}{|c|}{ Threshold (unit) } \\
\hline FEF & $2057 \pm 536$ & $2008 \pm 633$ & $-48.9 \pm 285.4$ & \\
\hline SC & $2243 \pm 1175$ & $2428 \pm 1348$ & $185.0 \pm 587.9$ & \\
\hline \multicolumn{5}{|c|}{ Rate (unit/ms) } \\
\hline FEF & $9.66 \pm 2.32$ & $10.04 \pm 2.99$ & $0.37 \pm 1.24$ & \\
\hline SC & $12.32 \pm 4.45$ & $12.18 \pm 4.96$ & $-0.14 \pm 1.95$ & \\
\hline \multicolumn{5}{|c|}{ Onset time (ms) } \\
\hline FEF & $42 \pm 39$ & $71 \pm 61$ & $29.6 \pm 23.8$ & * \\
\hline SC & $68 \pm 41$ & $58 \pm 41$ & $-9.5 \pm 20.9$ & \\
\hline
\end{tabular}

Data in the first two columns are mean \pm SD. ${ }^{*} p<0.05,{ }^{* *} p<0.01$.

The difference in onset time was smaller for the SC neurons than for the FEF neurons. However, the difference in RT was also smaller during sessions when the SC data were collected than in sessions when the FEF data were collected. Thus, the qualitative patterns of behavioral and neural data were the same in SC and FEF datasets; both showed slowing of RT after canceled stop trials and both showed a corresponding shift in the onset of accumulation and not in baseline, rate of accumulation, or threshold. The quantitative differences between the FEF and SC datasets should not obscure the qualitative similarity in the relationships. Indeed, the combination of data across laboratories and neural structures increases confidence in the reliability of the general conclusions.

\section{Ballistic accumulator model}

The activity of movement neurons in FEF and SC has been described as instantiating a stochastic accumulation process (Carpenter and Williams, 1995; Hanes and Schall, 1996; Schall, 2004; Boucher et al., 2007; Ratcliff et al., 2007; Carpenter et al., 2009). To investigate this linking proposition further, we fit a linear ballistic accumulator model (Brown and Heathcote, 2008). The behavioral data consisted of the RT distributions of no-stopsignal trials preceded by either a canceled stop-signal trial or a no-stop-signal trial. The model was fit separately to the RTs from each of the 36 sessions during which the movement neuron data were being collected from the frontal eye fields or superior colliculus. RT on a simulated trial was given by the time at which threshold was reached plus a fixed ballistic movement time of 10 $\mathrm{ms}$; this interval corresponds to the time before saccade initiation when omnipause neurons in the brainstem release inhibition on the medium lead burst neurons (Scudder et al., 2002).

Table 3 lists the values of the rate, onset, and threshold, plus predicted RTs for the two trial sequences when all parameters

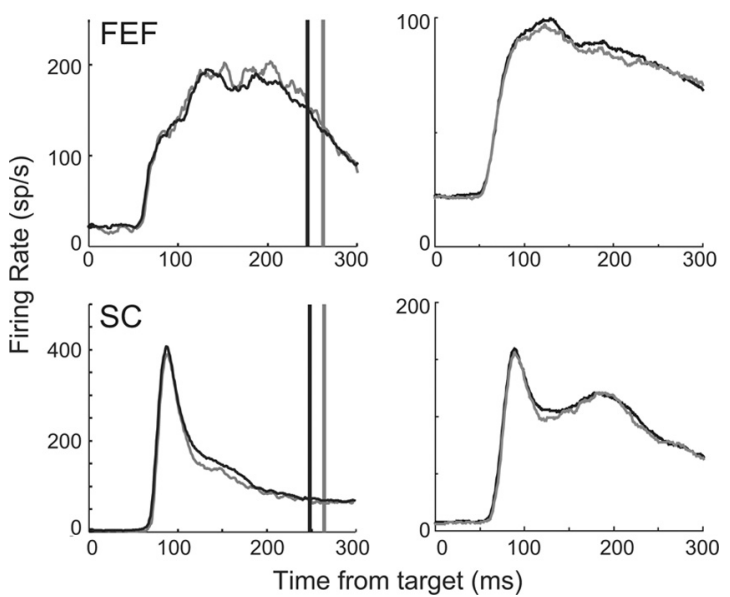

Figure 6. Visual responses in $\mathrm{FEF}$ (top) and SC (bottom) for representative neurons (left) and average (right) for no-stop-signal trials preceded by either no-stop-signal (black) or canceled (gray) trials. Average RT (vertical line) in the two trial sequences is indicated for the individual neurons.

were free to vary. This model accounted for the RTs and their difference between the two trial sequences. It fit the difference of RTs in the FEF data through a significant difference in the onset time with no significant difference in threshold or rate of accumulation. No significant difference was observed for the SC fits, but as discussed below, the RT difference for these data was rather small.

\section{Pattern of activity of visual neurons}

One plausible explanation for the change in onset time with the increase of RT after canceled stop trials is a strategic adjustment in a stage of processing preceding the movement neurons. One earlier stage strongly implicated in this task is encoding the location of the target, which is represented in the activity of visually responsive neurons in FEF and SC. If RT slowing resulted from a strategic adjustment in an earlier stage of processing, then we should see different response latencies or different discharge rates in visually responsive neurons on no-stop-signal trials preceded by canceled trials compared to no-stop-signal trials preceded by no-stop-signal trials. However, in both the FEF and SC data, we found no systematic differences in the latency or the magnitude of the evoked responses of visual neurons measured in the different trial sequences (Fig. 6, Table 1). These results are inconsistent with the hypothesis that the RT slowing observed in this task was due to an adaptive adjustment in visual processing preceding the movement neurons in FEF or SC.

\section{Discussion}

We found that a systematic elevation of RT after successful stopsignal trials was accomplished through a change in the time when 
the activity of movement neurons in FEF and SC first began to accumulate, and not through a change of threshold, baseline, or accumulation rate. We will argue that this new result provides new insights into the neural instantiation of stochastic accumulator models and the mechanisms through which executive control can be exerted.

\section{Relation to previous work on RT adjustments}

Numerous studies of speed-accuracy adjustments have been conducted with human participants but none using neurophysiological measures from macaque monkeys. Human brain imaging studies have implicated medial frontal cortical areas and the basal ganglia (e.g., Forstmann et al., 2008; see also Frank, 2006). ERP results obtained in various tasks including stop signal demonstrate changes both preceding and following the onset of the lateralized readiness potential (e.g., Osman et al., 2000; Rinkenauer et al., 2004; Upton et al., 2010). However, fMRI and ERP data provide insufficient temporal or spatial information to resolve alternative hypotheses about the mechanisms responsible for accomplishing the adjustment of RT that have been formulated through stochastic accumulator models of RT.

\section{Movement neurons and stochastic accumulation}

Stochastic accumulator models provide a powerful explanation of the random and systematic variation of RT in speeded response tasks (e.g., Ratcliff and Smith, 2004; Bogacz et al., 2006; Ratcliff and McKoon, 2008). We suggest that post-stop-signal slowing may rely on the same mechanisms as speed-accuracy adjustments. Typically, RT and choice probability are accounted for in terms of three parameters: baseline level before the accumulation begins, the rate of accumulation, and the threshold for terminating the accumulation process. A fourth parameter, residual processing time, is not considered formally part of the accumulation process. This residual time includes the time between the presentation of a stimulus and the beginning of accumulation of the decision variable (onset time) plus the time between terminating the accumulation and executing the response (motor time).

Stochastic accumulator models formulated to minimize errors and maximize rewards accomplish the adaptation through changes in the threshold to terminate accumulation (Bogacz et al., 2006; Nakahara et al., 2006; Simen et al., 2006; Ratcliff and McKoon, 2008). As yet, no model has proposed that the adaptation of RT involves changing the time when accumulation begins or the efferent delay time, although Verbruggen and Logan (2009) observed changes in onset in proactive adjustments of RT in the stop-signal task. The hypothesis that adjustment of RT is accomplished through changes in the threshold or rate of accumulation has not been tested directly by measuring those quantities in neurons that can instantiate the process described by stochastic accumulator models in monkeys optimizing RT to perform a task.

The neural instantiation of the processes described by stochastic accumulator models was elucidated with the discovery that responses are initiated when the random accumulation of activity in movement neurons in FEF reaches a fixed threshold (Hanes and Schall, 1996). Several lines of evidence support this linking proposition (Schall, 2004). (1) The temporal pattern of the stochastic accumulation process resembles the form of movement neuron activity (Hanes and Schall, 1996). (2) Models of stochastic accumulation processes map onto the temporal pattern of activity of movement neurons for tasks requiring categorization of a single stimulus (Ratcliff et al., 2007) and for visual search
(Purcell et al., 2010). (3) The activity of movement neurons in FEF and SC modulates in a manner sufficient to control the initiation of eye movements in the stop-signal task. Saccades are produced despite the stop signal if the activity of these neurons reaches threshold too rapidly, and saccades are not produced on stop-signal trials when the activity of these movement neurons is inhibited before it reaches threshold (Hanes et al., 1998; Paré and Hanes, 2003). (4) Behavior in the stop-signal paradigm can be modeled very accurately as a race between stochastic GO and STOP processes (Logan and Cowan, 1984), and a neural network model with interacting GO and STOP accumulator units replicates the time course of the activation of the movement neurons (Boucher et al., 2007; Lo et al., 2009; Wong-Lin et al., 2010). Thus, neural measures of the onset of accumulation, baseline discharge rate before the onset, rate of accumulation, and threshold can be identified with the corresponding parameters in stochastic accumulator models.

Evidence to evaluate this linking proposition was sought by fitting a ballistic accumulator model to the performance. A significant change in the onset parameter accounted for the systematic difference in RT regardless of the architecture. Thus, the pattern of activation of the movement neurons is consistent with the form of activation of stochastic and ballistic accumulators fit to the behavioral data. The data obtained in this experiment provide evidence that subjects adapt by changing the onset time of the accumulation process, not the threshold, as commonly believed.

\section{Visual processing and strategic adjustment}

One could argue that the FEF and SC movement neurons are not part of the decision process that is adjusted strategically because the accumulation is delayed by preceding visual processing. In fact, when visual search is less efficient, resulting in systematically longer RT, the movement neurons in FEF exhibit a delay in accumulation (Woodman et al., 2008) that corresponds to the delay in time when the FEF visual neurons select the less salient target (Sato et al., 2001). It is well known that the responses of visual cortical neurons have longer latency and lower magnitude for stimuli with lower contrast or a nonpreferred feature. However, in the current task the stimulus on every trial was a single suprathreshold spot of light. While we do not have data from extrastriate cortical areas, there is no reason to believe that any of these neurons respond systematically differently to the target stimulus in different trial contexts. Visual neurons in FEF and SC receive converging inputs from several areas in which perceptual and cognitive processing occurs (Lui et al., 1995; Schall et al., 1995; May, 2006). Thus, strategic adjustments of visual processing should be evident in the activity of the visually responsive neurons in FEF and SC. No changes were seen. This seems to rule out the possibility that the changes in RT following a stop signal were produced in earlier visual processes.

The semantic, logical, and mechanistic distinctions between categorization ("decide that") and response selection ("decide to") (Schall, 2001) suggests that a sequence of stochastic accumulators instantiates successive stages of processing. If so, then different accumulators will limit performance in different task conditions depending on the difficulty of categorization and response preparation. In the saccade stop-signal task, visual encoding and stimulus-response mapping were simple and direct, so response preparation must be the rate-limiting process. We recently demonstrated how movement neuron activity can be understood as a gated accumulation of target salience evidence from FEF visual neurons (Purcell et al., 2010). We are now exploring 
the possibility that a gate between visually responsive neurons and movement neurons can be a source of executive control. Increasing the gate that sensory evidence must exceed to merit accumulation will delay the onset of accumulation of movement neuron activity and thereby increase RT. It can also increase accuracy, because delaying the initiation of a response allows the categorization process more time to arrive at correct description.

\section{Sources of executive control}

Different sources of executive control have been described that can delay movement activity onset time by extending the holding period of the top-down control module as in the model of Lo et al. (2009). First, the role of medial frontal cortex in the endogenous control of action is beyond dispute (e.g., Ridderinkhof et al., 2004; Nachev et al., 2008; Rushworth, 2008). Indeed, neurons in the supplementary eye field of macaques performing saccade countermanding produce activity that predicts but does not control whether monkeys will cancel successfully (Stuphorn et al., 2010; see also Chen et al., 2010; Scangos and Stuphorn, 2010). Also, weak electrical stimulation of sites in the supplementary eye field improves stop-signal task performance by increasing RT (Stuphorn and Schall, 2006).

Second, previous work has also suggested memory processes are involved in response adjustments in the stop-signal task (Verbruggen and Logan, 2008a,b; Verbruggen et al., 2008). Such a role for memory is consistent with the observation that the amplitude of the P3b event-related potential component is modulated with stop trial history (Upton et al., 2010) and that modulation in this ERP component has been linked to memory processes (see for a review Polich, 2007). The posterior source of this component suggests contributions from parietal or temporal cortex. Whatever their source, adaptive signals from these cortical areas could in turn be facilitated and effected through the basal ganglia circuit, so that premature accumulation of movement activity is prevented (Frank, 2006).

In summary, these new results indicate that accumulation threshold is not the exclusive locus of strategic control and demonstrate how the stochastic process producing responses can enact strategic adjustments to optimize performance. This result provides new insights into the neural instantiation of stochastic accumulator models and the mechanisms through which executive control is exerted.

\section{References}

Bichot NP, Thompson KG, Chenchal Rao S, Schall JD (2001) Reliability of macaque frontal eye field neurons signaling saccade targets during visual search. J Neurosci 21:713-725.

Bogacz R, Brown E, Moehlis J, Holmes P, Cohen JD (2006) The physics of optimal decision making: a formal analysis of models of performance in two-alternative forced-choice tasks. Psychol Rev 113:700-765.

Boucher L, Palmeri TJ, Logan GD, Schall JD (2007) Inhibitory control in mind and brain: an interactive race model of countermanding saccades. Psychol Rev 114:376-397.

Brown JW, Hanes DP, Schall JD, Stuphorn V (2008) Relation of frontal eye field activity to saccade initiation during a countermanding task. Exp Brain Res 190:135-151.

Brown SD, Heathcote A (2008) The simplest complete model of choice response time: linear ballistic accumulation. Cogn Psychol 57:153-178.

Carpenter RH, Williams ML (1995) Neural computation of log likelihood in control of saccadic eye movements. Nature 377:59-62.

Carpenter RH, Reddi BA, Anderson AJ (2009) A simple two-stage model predicts response time distributions. J Physiol 587:4051-4062.

Chen X, Scangos KW, Stuphorn V (2010) Supplementary motor area exerts proactive and reactive control of arm movements. J Neurosci 30:14657-14675.

Dorris MC, Paré M, Munoz DP (1997) Neuronal activity in monkey supe- rior colliculus related to the initiation of saccadic eye movements. J Neurosci 17:8566-8579.

Emeric EE, Brown JW, Boucher L, Carpenter RH, Hanes DP, Harris R, Logan GD, Mashru RN, Paré M, Pouget P, Stuphorn V, Taylor TL, Schall JD (2007) Influence of history on countermanding performance in humans and macaque monkeys. Vision Res 47:35-49.

Forstmann BU, Dutilh G, Brown S, Neumann J, von Cramon DY, Ridderinkhof KR, Wagenmakers EJ (2008) Striatum and pre-SMA facilitate decision-making under time pressure. Proc Natl Acad Sci USA 105:17538-17542.

Frank MJ (2006) Hold your horses: a dynamic computational role for the subthalamic nucleus in decision making. Neural Netw 19:1120-1136.

Gold JI, Shadlen MN (2007) The neural basis of decision making. Annu Rev Neurosci 30:535-574.

Hanes DP, Schall JD (1996) Neural control of voluntary movement initiation. Science 274:427-430.

Hanes DP, Patterson WF 2nd, Schall JD (1998) The role of frontal eye field in countermanding saccades: visual, movement and fixation activity. J Neurophysiol 79:817-834.

Lo CC, Boucher L, Paré M, Schall JD, Wang XJ (2009) Proactive inhibitory control and attractor dynamics in countermanding action: a spiking neural circuit model. J Neurosci 29:9059-9071.

Logan GD, Cowan WB (1984) On the ability to inhibit thought and action: a theory of an act of control. Psychol Rev 91:295-327.

Lui F, Gregory KM, Blanks RH, Giolli RA (1995) Projections from visual areas of the cerebral cortex to pretectal nuclear complex, terminal accessory optic nuclei, and superior colliculus in macaque monkey. J Comp Neurol 363:439-460.

May PJ (2006) The mammalian superior colliculus: laminar structure and connections. Prog Brain Res 151:321-378.

Mazurek ME, Roitman JD, Ditterich J, Shadlen MN (2003) A role for neural integrators in perceptual decision making. Cereb Cortex 13:1257-1269.

Murthy A, Ray S, Shorter SM, Schall JD, Thompson KG (2009) Neural control of visual search by frontal eye field: effects of unexpected target displacement on visual selection and saccade preparation. J Neurophysiol 101:2485-2506.

Nachev P, Kennard C, Husain M (2008) Functional role of the supplementary and pre-supplementary motor areas. Nat Rev Neurosci 9:856-869.

Nakahara H, Nakamura K, Hikosaka O (2006) Extended LATER model can account for trial-by-trial variability of both pre- and post-processes. Neural Netw 19:1027-1046.

Nelder JA, Mead R (1965) A simple method for function minimization. Comp J 7:308-313.

Nelson MJ, Boucher L, Logan GD, Palmeri TJ, Schall JD (2010) Nonstationarity of saccade response time in stopping and stepping tasks. Atten Percept Psychophys 72:1913-1929.

Osman A, Lou L, Muller-Gethmann H, Rinkenauer G, Mattes S, Ulrich R (2000) Mechanisms of speed-accuracy tradeoff: evidence from covert motor processes. Biol Psychol 51:173-199.

Paré M, Hanes DP (2003) Controlled movement processing: superior colliculus activity associated with countermanded saccades. J Neurosci 23:6480-6489

Polich J (2007) Updating P300: an integrative theory of P3a and P3b. Clin Neurophysiol 118:2128-2148.

Purcell BA, Heitz RP, Cohen JY, Schall JD, Logan GD, Palmeri TJ (2010) Neurally constrained modeling of perceptual decision making. Psychol Rev 117:1113-1143.

Ratcliff R, McKoon G (2008) The diffusion decision model: theory and data for two-choice decision tasks. Neural Comput 20:873-922.

Ratcliff R, Smith PL (2004) A comparison of sequential sampling models for two-choice reaction time. Psychol Rev 111:333-367.

Ratcliff R, Tuerlinckx F (2002) Estimating parameters of the diffusion model: approaches to dealing with contaminant reaction times and parameter variability. Psychon Bull Rev 9:438-481.

Ratcliff R, Hasegawa YT, Hasegawa RP, Smith PL, Segraves MA (2007) Dual diffusion model for single-cell recording data from the superior colliculus in a brightness-discrimination task. J Neurophysiol 97:1756-1774.

Ray S, Pouget P, Schall JD (2009) Functional distinction between visuomovement and movement neurons in macaque frontal eye field during saccade countermanding. J Neurophysiol 102:3091-3100.

Ridderinkhof KR, Ullsperger M, Crone EA, Nieuwenhuis S (2004) The role of the medial frontal cortex in cognitive control. Science 306:443-447. 
Rieger M, Gauggel S (1999) Inhibitory after-effects in the stop signal paradigm. Br J Psychol 90:509-518.

Rinkenauer G, Osman A, Ulrich R, Muller-Gethmann H, Mattes S (2004) On the locus of speed-accuracy trade-off in reaction time: inferences from the lateralized readiness potential. J Exp Psychol Gen 133:261-282.

Rowan T (1990) Functional stability analysis of numerical algorithms, PhD thesis, University of Texas at Austin.

Rushworth MF (2008) Intention, choice, and the medial frontal cortex. Ann N Y Acad Sci 1124:181-207.

Sato TR, Schall JD (2003) Effects of stimulus-response compatibility on neural selection in frontal eye field. Neuron 38:637-648.

Sato T, Murthy A, Thompson KG, Schall JD (2001) Search efficiency but not response interference affects visual selection in frontal eye field. Neuron 30:583-591.

Scangos KW, Stuphorn V (2010) Medial frontal cortex motivates but does not control movement initiation in the countermanding task. J Neurosci 30:1968-1982.

Schall JD (2001) Neural basis of deciding, choosing and acting. Nat Rev Neurosci 2:33-42.

Schall JD (2004) On building a bridge between brain and behavior. Annu Rev Psychol 55:23-50.

Schall JD, Boucher L (2007) Executive control of gaze by the frontal lobes. Cogn Affect Behav Neurosci 7:396-412.

Schall JD, Morel A, King DJ, Bullier J (1995) Topography of visual cortex connections with frontal eye field in macaque: convergence and segregation of processing streams. J Neurosci 15:4464-4487.

Scudder CA, Kaneko CS, Fuchs AF (2002) The brainstem burst generator for saccadic eye movements: a modern synthesis. Exp Brain Res 142:439-462.

Simen P, Cohen JD, Holmes P (2006) Rapid decision threshold modulation by reward rate in a neural network. Neural Netw 19:1013-1026.
Smith PL, Ratcliff R (2004) Psychology and neurobiology of simple decisions. Trends Neurosci 27:161-168.

Sparks DL (1978) Functional properties of neurons in the monkey superior colliculus: coupling of neuronal activity and saccade onset. Brain Res 156:1-16.

Stuphorn V, Schall JD (2006) Executive control of countermanding saccades by the supplementary eye field. Nat Neurosci 9:925-931.

Stuphorn V, Brown JW, Schall JD (2010) Role of supplementary eye field in saccade initiation: executive, not direct, control. J Neurophysiol 103:801-816.

Upton DJ, Enticott PG, Croft RJ, Cooper NR, Fitzgerald PB (2010) ERP correlates of response inhibition after-effects in the stop signal task. Exp Brain Res 206:351-358.

Verbruggen F, Logan GD (2008a) Long-term aftereffects of response inhibition: memory retrieval task goals and cognitive control. J Exp Psychol Hum Percept Perform 34:1229-1235.

Verbruggen F, Logan GD (2008b) Automatic and controlled response inhibition: associative learning in the go/no-go and stop-signal paradigms. J Exp Psychol Gen 137:649-672.

Verbruggen F, Logan GD (2008c) Response inhibition in the stop-signal paradigm. Trends Cogn Sci 12:418-424.

Verbruggen F, Logan GD (2009) Proactive adjustments of response strategies in the stop-signal paradigm. J Exp Psychol Hum Percept Perform 35:835-854.

Verbruggen F, Logan GD, Liefooghe B, Vandierendonck A (2008) Aftereffects of response inhibition: repetition priming or between-trial control adjustments? J Exp Psychol Human 34:413-426.

Wong-Lin K, Eckhoff P, Holmes P, Cohen JD (2010) Optimal performance in a countermanding saccade task. Brain Res 1318:178-187.

Woodman GF, Kang MS, Thompson K, Schall JD (2008) The effect of visual search efficiency on response preparation: neurophysiological evidence for discrete flow. Psychol Sci 19:128-136. 